\title{
CFBDS J005910.90-011401.3: reaching the T-Y brown dwarf transition? ${ }^{\star}$
}

\author{
P. Delorme ${ }^{1}$, X. Delfosse ${ }^{1}$, L. Albert ${ }^{2}$, E. Artigau ${ }^{3}$, T. Forveille ${ }^{1,4}$, C. Reylé ${ }^{5}$, F. Allard ${ }^{6}$, D. Homeier ${ }^{7}$, \\ A. C. Robin $^{5}$, C. J. Willott ${ }^{8}$, M. C. Liu $^{4}{ }^{\star \star \star}$, and T. J. Dupuy ${ }^{4}$ \\ ${ }^{1}$ Laboratoire d'Astrophysique de Grenoble, Observatoire de Grenoble, Université Joseph Fourier, CNRS, UMR 5571 Grenoble, \\ France \\ e-mail: Philippe.Delorme@obs.ujf-grenoble.fr \\ 2 Canada-France-Hawaii Telescope Corporation, 65-1238 Mamaloha Highway, Kamuela, HI 96743, USA \\ ${ }^{3}$ Gemini Observatory, Southern Operations Center, Association of Universities for Research in Astronomy, Inc., Casilla 603, \\ La Serena, Chile \\ 4 Institute for Astronomy, 2680 Woodlawn Drive, Honolulu, HI 96822-1839, USA \\ 5 Observatoire de Besançon, Institut UTINAM, University of Franche-Comté, CNRS-UMR 6213, BP 1615, 25010 Besançon Cedex, \\ France \\ ${ }^{6}$ Centre de Recherche Astrophysique de Lyon, UMR 5574 CNRS, Université de Lyon, École Normale Supérieure de Lyon, \\ 46 allée d'Italie, 69364 Lyon Cedex 07, France \\ 7 Institut für Astrophysik, Georg-August-Universität, Friedrich-Hund-Platz 1, 37077 Göttingen, Germany \\ 8 Physics Department, University of Ottawa, 150 Louis Pasteur, MacDonald Hall, Ottawa, ON K1N 6N5, Canada
}

Received 21 December 2007 / Accepted 5 March 2008

\section{ABSTRACT}

\begin{abstract}
Aims. We report the discovery of CFBDS J005910.90-011401.3 (hereafter CFBDS0059), the coolest brown dwarf identified to date. Methods. We found CFBDS0059 using $i^{\prime}$ and $z^{\prime}$ images from the Canada-France-Hawaii Telescope (CFHT), and present optical and near-infrared photometry, Keck laser-guide-star adaptive optics imaging, and a complete near-infrared spectrum, from 1.0 to $2.2 \mu \mathrm{m}$. Results. A side-to-side comparison of the near-infrared spectra of CFBDS0059 and ULAS J003402.77-005206.7 (hereafter ULAS0034), previously the coolest known brown dwarf, indicates that CFBDS0059 is $\sim 50 \pm 15 \mathrm{~K}$ cooler. We estimate a temperature of $T_{\text {eff }} \sim 620 \mathrm{~K}$ and gravity of $\log g \sim 4.75$. Evolutionary models translate these parameters into an age of $1-5$ Gyr and a mass of 15-30 $M_{\text {Jup }}$. We estimate a photometric distance of $\sim 13 \mathrm{pc}$, which puts CFBDS0059 within easy reach of accurate parallax measurements. Its large proper motion suggests membership in the older population of the thin disk. The spectra of both CFBDS0059 and ULAS J0034 show probable absorption by a wide ammonia band on the blue side of the $H$-band flux peak. If, as we expect, that feature deepens further for still lower effective temperatures, its appearance will become a natural breakpoint for the transition between the T spectral class and the new Y spectral type. Together, CFBDS0059 and ULAS J0034 would then be the first Y0 dwarfs.
\end{abstract}

Key words. techniques: spectroscopic - surveys - stars: atmospheres - infrared: stars - stars: low-mass, brown dwarfs

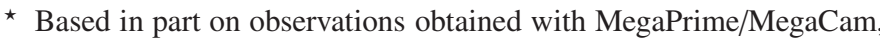
a joint project of CFHT and CEA/DAPNIA, at the Canada-FranceHawaii Telescope (CFHT) which is operated by the National Research Council (NRC) of Canada, the Institut National des Sciences de l'Univers of the Centre National de la Recherche Scientifique (CNRS) of France, and the University of Hawaii. This work is based in part on data products produced at TERAPIX and the Canadian Astronomy Data Centre as part of the Canada-France-Hawaii Telescope Legacy Survey, a collaborative project of NRC and CNRS. Also based on observations obtained at the Gemini Observatory, which is operated by the Association of Universities for Research in Astronomy, Inc., under a cooperative agreement with the NSF on behalf of the Gemini partnership: the National Science Foundation (United States), the Science and Technology Facilities Council (United Kingdom), the National Research Council (Canada), CONICYT (Chile), the Australian Research Council (Australia), CNPq (Brazil) and SECYT (Argentina) under programs GN-2007A-Q-201 and GN-2007B-Q-3. Also based on observations made with ESO Telescopes at the La Silla Observatory under programmes 078.C-0629 and 078.A-0651. Some of the data presented herein were obtained at the W. M. Keck Observatory, which is operated as a scientific partnership among the California Institute of Technology, the University of California, and the National
}

\section{Introduction}

Observed stellar and substellar atmospheres cover a continuum of physical conditions from the hottest stars $(\sim 100000 \mathrm{~K})$ to the coolest known brown dwarfs (previously ULAS J003402.77005206.7 (hereafter ULAS0034), >T8 Warren et al. 2007). There remains however a sizeable temperature gap between the $600-700 \mathrm{~K}$ ULAS0034 and the $\sim 100 \mathrm{~K}$ giant planets of the Solar System. Many of the currently known extrasolar planets populate this temperature interval, characterized by complex atmospheric physics: matter and radiation in these cold, dense, and turbulent atmospheres couple into a very dynamical mix, where molecules and dust form and dissipate. Current atmosphere models are rather uncertain in this unexplored temperature range and they will significantly benefit from observational constraints. Two major physical transitions are expected to occur between $\sim 700 \mathrm{~K}$ and $\sim 400 \mathrm{~K}$ and strongly alter the emergent near-infrared spectra (Burrows et al. 2003): $\mathrm{NH}_{3}$ becomes an

Aeronautics and Space Administration. The Observatory was made possible by the generous financial support of the W. M. Keck Foundation. $\star \star$ Alfred P. Sloan Research Fellow. 
abundant atmospheric constituent and its near-infrared bands become major spectral features, and water clouds form and deplete $\mathrm{H}_{2} \mathrm{O}$ from the gas phase. The corresponding near-infrared spectral changes are likely to be sufficiently drastic that the creation of a new spectral type will be warranted (Kirkpatrick 2000). Kirkpatrick et al. (1999); Kirkpatrick (2000) reserved the "Y" letter for the name of that putative new spectral type.

To help fill this temperature gap, we conduct the Canada France Brown Dwarf Survey (CFBDS, Delorme et al. 2008), which uses MegaCam (Boulade et al. 2003) $i^{\prime}$ and $z^{\prime}$ images to select very cool brown dwarfs (and high redshift quasars) on their very red $i^{\prime}-z^{\prime}$ colour. We present here our coolest brown dwarf discovery to date, CFBDS J005910.90-011401.3 (hereafter CFBDS0059), a > T8 dwarf with evidence of near-infrared $\mathrm{NH}_{3}$ absorption. Section 2 describes its discovery, and presents our follow-up observations: $i^{\prime}, z^{\prime}, Y, J, H$ and $K$ s photometry and astrometry of CFBDS0059 and (as a reference) ULAS0034, laser guide star adaptive optics imaging and a near infrared spectrum of the new brown dwarf. Section 3 discusses the kinematics and the dynamical population membership of CFBDS0059. Section 4 compares the spectrum of CFBDS0059 with those of Gl570D (T7.5), 2MASS J0415-09 (T8) and ULAS0034, and in the light of synthetic spectra uses that comparison to determine its effective temperature, gravity and metallicity. We also examine in that section the new spectral features which appear below $700 \mathrm{~K}$, in particular an $\mathrm{NH}_{3}$ band, and discuss new spectral indices for spectral classification beyond T8. Finally, Sect. 5 summarizes our findings and sketches our near-future plans.

\section{CFBDS}

Field ultracold brown dwarfs are intrinsically very faint, and as a result they can only be identified in sensitive wide-field imaging surveys. They are most easily detected in the near infrared, and one could thus naively expect them to be most easily identified in that wavelength range. Brown dwarf spectra however very much differ from a black body, and their considerable structure from deep absorption lines and bands produces broad-band pure nearinfrared colours that loop back to the blue. At modest signal to noise ratios, those colours are not very distinctive. Brown dwarfs are therefore more easily recognized by including at least one photometric band under $1 \mu \mathrm{m}$. At those shorter wavelengths their spectra have extremely steep spectral slopes, and the resulting very red $i^{\prime}-z^{\prime}$ and $z^{\prime}-J$ colours easily stand out.

As discussed in detail in Delorme et al. (2008), the CFBDS survey brown dwarf identification is a two-step process:

- we first select brown dwarfs candidates on their red $i^{\prime}-z^{\prime}$ colour in MegaCam images which cover several hundred square degrees;

- J-band pointed observations of these candidates then discriminate actual brown dwarfs from artefacts, and astrophysical contaminants.

The $i^{\prime}-z^{\prime}$ selection takes advantage of the wide field of the MegaCam camera ${ }^{1}$ on the $3.6 \mathrm{~m}$ CFHT telescope, and of the trove of deep observational material obtained with that instrument. We rely on existing $i^{\prime}$ images from the Very Wide component of the Canada France Hawaii Telescope Legacy Survey (CFHTLS-VW) and, for different fields, on existing $z^{\prime}$ images from the Red sequence Cluster Survey 2 (RCS2). We then match those with either new $z^{\prime}$ or new $i^{\prime}$ exposures to obtain $i^{\prime}$ and

\footnotetext{
1 http://www-dapnia.cea.fr/Sap/Phys/Sap/Activites/ Projets/Megacam
}

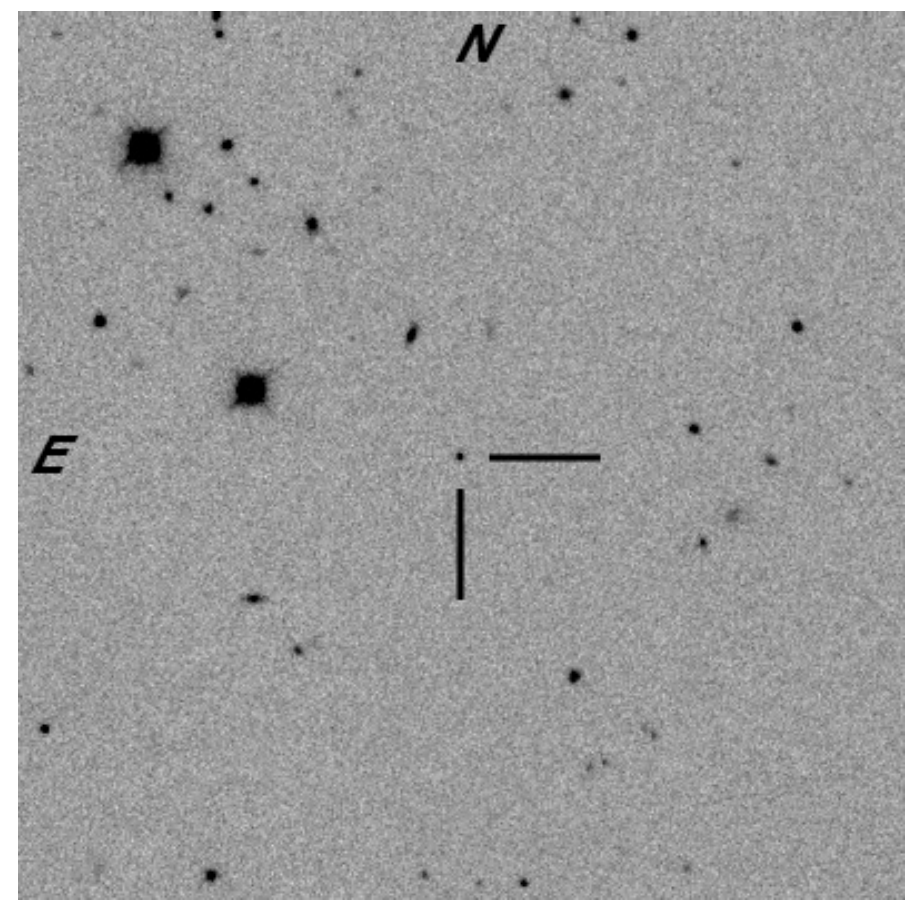

Fig. 1. $K_{\mathrm{S}}$-band finder chart for CFBDS0059. The field of view is 2 by 2 arcmin.

$z^{\prime}$ pairs. The two parent surveys also obtain $g^{\prime}$ and $r^{\prime}$ images, which for the RCS2 survey are contemporaneous with the $z^{\prime}$ exposure. We don't use those as primary selection tools, but the contemporaneous exposures from the RCS2 survey provide a welcome check that an apparently red source was not, instead, a variable brighter at all wavelenghts at the $z^{\prime}$ epochs. Since all our fields have low galactic extinction, the only other astrophysical point sources with a similarly red $i^{\prime}-z^{\prime}$ are quasars at redshifts of $z \approx 6$, which represent the other motivation of our program (e.g. Willott et al. 2007). We discriminate between quasars and brown dwarfs with $J$-band photometry obtained on several 2 to $4 \mathrm{~m}$-class telescopes (Delorme et al. 2008, for details). These targeted follow-up observations also reject a number of remaining unflagged artefacts and they provide a refined spectral type estimate, thanks to the much higher signal-to-noise ratio which we typically achieve on $z^{\prime}-J$ than on $i^{\prime}-z^{\prime}$.

\section{Observations}

We first identified CFBDS0059 as a brown dwarf candidate when comparing a 360 s RCS2 $z^{\prime}$ exposure from 2005 December 27 with a $500 \mathrm{~s}$ CFBDS $i^{\prime}$ exposure from 2006 August 31. CFBDS0059 is undetected in the $i^{\prime}$ image to $i_{\mathrm{AB}}^{\prime}=$ $25.2(5 \sigma)$, in spite of a strong $z^{\prime}$ detection $\left(z_{\mathrm{AB}}^{\prime}=21.93 \pm 0.05\right)$. The RCS2 survey obtains contemporaneous $g^{\prime}, r^{\prime}$ and $z^{\prime}$ images, and we checked the $g^{\prime}$ and $r^{\prime}$ exposures for a counterpart. These images, which were obtained within 50 min of the $z^{\prime}$ observation, show no object at the position of CFBDS0059. This essentially excludes that the $z^{\prime}$ detection was due to a variable or slowly moving object with neutral colours. The $i_{\mathrm{AB}}^{\prime}-z_{\mathrm{AB}}^{\prime}>3.2(5 \sigma)$ lower limit was thus secure, and made CFBDS0059 a very strong candidate for follow-up. 
Table 1. Wide band $i_{\mathrm{AB}}^{\prime}$ and $z_{\mathrm{AB}}^{\prime}$ CFHT photometry measured with Megacam, MKO-system $Y, J, H$, and $K_{\mathrm{s}}$ measured with WIRCam and associated colours. For comparison we include previous $Y, J, H$, and $K$ photometry of ULAS0034.

\begin{tabular}{|c|c|c|c|c|c|c|c|}
\hline \multirow[t]{2}{*}{ Target } & \multicolumn{7}{|c|}{ Spectral band } \\
\hline & $i_{\mathrm{AB}}^{\prime}$ & $z_{\mathrm{AB}}^{\prime}$ & Y & $J$ & $H$ & $K_{s}$ & $K$ \\
\hline CFBDS0059 & $>25.17 \pm 0.03$ & $21.93 \pm 0.05$ & $18.82 \pm 0.02$ & $18.06 \pm 0.03$ & $18.27 \pm 0.05$ & $18.63 \pm 0.05$ & - \\
\hline ULAS0034 & $>25.14 \pm 0.04$ & $22.11 \pm 0.06$ & $19.12 \pm 0.03$ & $18.21 \pm 0.03$ & $18.49 \pm 0.05$ & $18.33 \pm 0.05$ & - \\
\hline ULASO034 $^{1}$ & - & - & $18.90 \pm 0.10$ & $18.15 \pm 0.03$ & $18.49 \pm 0.04$ & - & $18.48 \pm 0.05$ \\
\hline & $z_{\mathrm{AB}}^{\prime}-Y$ & $Y-J$ & $J-H$ & $H-K \mathrm{~s}$ & $H-K$ & & \\
\hline CFBDS0059 & $3.11 \pm 0.05$ & $0.76 \pm 0.04$ & $-0.21 \pm 0.06$ & $-0.36 \pm 0.07$ & - & & \\
\hline ULAS0034 & $2.99 \pm 0.07$ & $0.91 \pm 0.04$ & $-0.28 \pm 0.06$ & $0.16 \pm 0.07$ & - & & \\
\hline ULAS0034 ${ }^{1}$ & - & $0.75 \pm 0.10$ & $-0.34 \pm 0.05$ & - & $0.01 \pm 0.07$ & & \\
\hline
\end{tabular}

${ }^{1}$ Photometry from Warren et al. (2007).

\subsection{J-band photometry and near-infrared spectroscopy}

Our initial $J$-band imaging consists of five 20-s dithered exposures with the SOFI near-infrared camera on the ESO NTT telescope at La Silla on 2006 November 12. As discussed below, the photometric system of that instrument is non-standard. We used a modified version of the jitter utility within the ESO Eclipse package (Devillard 1997) to subtract the background and coadd the five exposures. We extracted photometry from the resulting image using PSF fitting within Source Extractor (Bertin \& Arnouts (1996) and Bertin \& Delorme, in preparation) and obtain $J_{\text {Vega }}=18.11 \pm 0.06$. The resulting $z_{\mathrm{AB}}-J_{\mathrm{AB}}=3.0$ colour confirmed CFBDS0059 as a strong very late-T dwarf candidate and we triggered $H$-band spectroscopic observations with NIRI (Hodapp et al. 2003) at Gemini-North. Those were obtained in queue mode on 2007 July 30 and immediately confirmed the very cool nature of CFBDS0059. We then requested $J$ and $K$-band spectroscopy with the same instrument, which was obtained on 2007 September 1.

All spectra were obtained through a $\sim 0.7^{\prime \prime}$-wide slit, which produces a resolving power of $\frac{\lambda}{\Delta \lambda} \sim 500$. The object is dithered along the slit. The $H$-band spectrum is the sum of 16300 -s integrations, while the $Y+J$ and $K$ band spectra each are the sum of 9300 -s integrations. Consecutive image pairs are pair subtracted, flat fielded using a median combined spectral flat and corrected for both spectral and spatial distortions. Spectra are extracted using a positive and a negative extraction box matched to the trace profile. A first wavelength calibration was obtained with argon lamp arc spectra taken at the end of the sequence, and the wavelength scale was then fine tuned to match the atmospheric $\mathrm{OH}-$ lines. Individual spectra extracted from image pairs were then normalized and median combined into final spectra. Per-pixel S/N of 25, 40 and 5 where achieved on the $J, H$ and $K$-band peaks respectively. For all 3 wavelength settings the A0 star HIP10512 was observed immediately after the science observations to calibrate the instrumental spectral response and the telluric transmission.

\subsection{Additional near-infrared and optical photometry}

The $J$ filter of the SOFI camera on the NTT has a quite nonstandard bandpass, for which the large colour corrections that result from the strong structrure in $T$ dwarf spectra (e.g. Stephens \& Leggett 2004) have not been fully characterized. To compare the spectral energy distribution (SEDs) of CFBDS0059 and ULAS0034 (Warren et al. 2007) we therefore prefered to obtain additional near-infrared wide-band photometry with WIRCam (Puget et al. 2004) on CFHT, which uses standard Mauna Kea Observatory infrared filters (Simons \& Tokunaga 2002; Tokunaga et al. 2002; Tokunaga \& Vacca 2005, MKO
Table 2. Astrometry of CFBDS0059 (Epoch: August 5th, 2007).

\begin{tabular}{llccc}
\hline \hline $\begin{array}{l}\text { RA(J2000) } \\
\text { hh:mm:ss }\end{array}$ & $\begin{array}{l}\text { Dec(J2000) } \\
\text { dd:mm:ss }\end{array}$ & \multicolumn{2}{c}{$\mu_{\alpha} \quad$ $/ \mathrm{yr}$} & $\mu_{\delta}$ \\
\hline 00:59:10.903 & $-01: 14: 01.13$ & $0.94 \pm 0.06$ & $0.18 \pm 0.06$ \\
\hline
\end{tabular}

system). The observations (2007 August 1st and 5th) used dithering patterns of $\sim 60$ arcsec amplitude for total (respectively individual) exposure times of 300 (60), 150 (30), 300 (15) and 720 (20) seconds for the $Y, J, H$ and $K_{\mathrm{s}}$ bands. The skies were photometric and the seeing varied between 0.8 and $1.0^{\prime \prime}$.

Table 1 summarizes the magnitudes of CFBDS0059 and ULAS0034 in all available bands. The WIRCam photometry of ULAS0034 agrees with the Warren et al. (2007) measurements within better than $1 \sigma$ for $H$ band and within $1.5 \sigma$ for $J$. The Warren et al. (2007) $K$-band measurement used a $K$ filter, while our WIRCam measurement uses the narrower and bluer $K$ s filter. The 0.15 mag difference between these two observations is approximately consistent with the Stephens \& Leggett (2004) prediction for the effect of these different filter bandpasses at late-T spectral types. Similarly, the better short-wavelength quantum efficiency of the WIRCam detector can qualitatively explain the 0.2 mag $(2 \sigma)$ discrepancy between our $Y$ photometry and the Warren et al. (2007) WFCam measurement. The near-IR colours of the two brown dwarfs are similar, except $H-K \mathrm{~s}$ which is $\sim 0.5$ mag bluer for CFBDS0059 than for ULAS0034. We will interpret the implications of this low $K$ s flux when we examine the near-infrared spectrum.

\subsection{Astrometry}

CFBDS0059 and ULAS0034 are serendipitously just 6.3 degrees apart on the sky, and at similar photometric distances from Earth since they have similar spectral types and apparent magnitudes, and we initially entertained the idea that they might, perhaps, be part of a common moving group. The proper motion of CFBDS0059 however, measured between our 2005 Megacam $z^{\prime}$ and 2007 WIRCam $K_{\mathrm{s}}$ images, and uncorrected for its parallactic motion, is $\mu_{\alpha}=+0.94 \pm 0.06^{\prime \prime} / \mathrm{yr}, \mu_{\delta}=$ $+0.18 \pm 0.06^{\prime \prime} / \mathrm{yr}$ (Table 2 ). ULAS0034 on the other hand moves by $\mu_{\alpha}=-0.12 \pm 0.05^{\prime \prime} / \mathrm{yr}$ and $\mu_{\delta}=0.35 \pm 0.005^{\prime \prime} / \mathrm{yr}$ (Warren et al. 2007). The two proper motions are thus sufficiently different that the two brown dwarfs are clearly unrelated. We checked for main sequence common proper motion companions to CFBDS0059, which would have provided welcome age and metallicity constraints (e.g. Scholz et al. 2003) but did not find any match within a 10 arcmin radius. 


\subsection{Keck laser guide star adaptive optics imaging}

To search for binarity, we imaged CFBDS0059 on 16 January 2008 UT using the laser guide star adaptive optics (LGS AO) system (van Dam et al. 2006; Wizinowich et al. 2006) of the 10-m Keck II Telescope on Mauna Kea, Hawaii. Conditions were photometric with better than average seeing. We used the facility IR camera NIRC2 with its narrow field-of-view camera, which produces an image scale of $9.963 \pm 0.011 \mathrm{mas} /$ pixel (Pravdo et al. 2006) and a 10.2" $\times 10.2^{\prime \prime}$ field of view. The LGS provided the wavefront reference source for AO correction, with the exception of tip-tilt motion. The LGS brightness was equivalent to a $V \approx 9.8 \mathrm{mag}$ star, as measured by the flux incident on the AO wavefront sensor. Tip-tilt aberrations and quasi-static changes in the image of the LGS as seen by the wavefront sensor were measured contemporaneously with a second, lower-bandwidth wavefront sensor monitoring the $R=14.6$ mag field star USNO-B1.0 0887-0010532 (Monet et al. 2003), located $32^{\prime \prime}$ away from CFBDS0059. The sodium laser beam was pointed at the centre of the NIRC2 field-of-view for all observations.

We obtained a series of dithered images, offsetting the telescope by a few arcseconds, with a total integration time of $1080 \mathrm{~s}$. We used the $\mathrm{CH} 4 \mathrm{~s}$ filter, which has a central wavelength of $1.592 \mu \mathrm{m}$ and a width of $0.126 \mu \mathrm{m}$. This filter is positioned near the $H$-band flux peak emitted by late-T dwarfs. The images were reduced in a standard fashion. We constructed flat fields from the differences of images of the telescope dome interior with and without continuum lamp illumination. Then we created a master sky frame from the median average of the biassubtracted, flat-fielded images and subtracted it from the individual images. Images were registered and stacked to form a final mosaic, with a full-width at half-maximum of $0.09^{\prime \prime}$ and a Strehl ratio of 0.05 . No companions were clearly detected in a $6^{\prime \prime} \times 6^{\prime \prime}$ region centred on CFBDS0059.

We determined upper limits from the direct imaging by first smoothing the final mosaic with an analytical representation of the PSF's radial profile, modeled as the sum of multiple gaussians. We then measured the standard deviation in concentric annuli centred on the science target, normalized by the peak flux of the targets, and adopted $10 \sigma$ as the flux ratio limits for any companions. These limits were verified with implantation of fake companions into the image using translated and scaled versions of the science target.

Figure 2 presents the final upper limits on any companions. We employed the "COND" models of Baraffe et al. (2003) to convert the limits into companion masses, for assumed ages of 1 and $5 \mathrm{Gyr}$ and a photometric distance estimate of $13 \mathrm{pc}$. We assumed any cooler companions would have similar $(\mathrm{CH} 4 \mathrm{~s}-\mathrm{H})$ colours to CFBDS0059.

\section{Kinematics}

We estimate a spectrophotometric distance for CFBDS0059 by adopting $M_{J}=17.5 \pm 0.5$, based on an approximate $\mathrm{T} 9 / \mathrm{Y} 0$ spectral type (discussed below) and on an extrapolation of the $M_{J}$ versus spectral type relation of Knapp et al. (2004) beyond T8 (2MASSJ0415 - 0935, hereafter 2M0415). This extrapolation is consistent with the Chabrier et al. (2000) models which predict $\Delta J \sim 1.0$ between brown dwarfs of $T_{\text {eff }} \sim 750 \mathrm{~K}$ and $625 \mathrm{~K}$ (like 2M0415 and CFBDS0059). The resulting $d=13 \pm 5 \mathrm{pc}$ has significant systematic uncertainties, because spectral typing beyond T8 is just being defined, and especially because the linear $1 \mathrm{mag} / \mathrm{subtype}$ decline seen at earlier subtypes may not continue

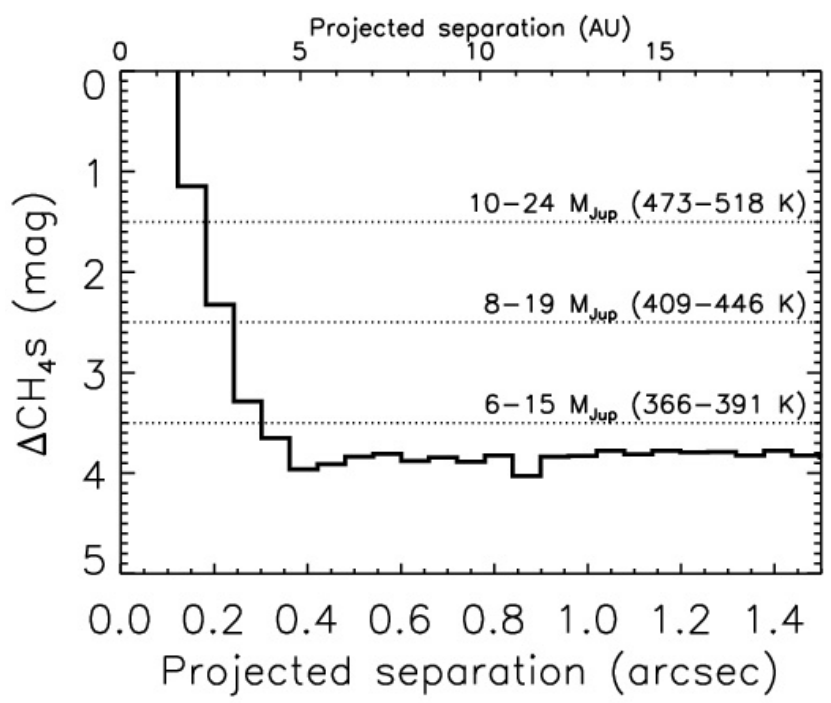

Fig. 2. Adaptive Optic detections limits as a function of the separation from CFBDS0059. Dashed lines show the equivalent mass and temperature for a given contrast. The first value assumes an age of $1 \mathrm{Gyr}$ and the second an age of 5 Gyr.

beyond T8. The adaptive optics observations exclude any companion of similar luminosity beyond 1.2 AU, but CFBDS0059 could still of course be a tighter binary. Its small distance fortunately puts CFBDS0059 within easy reach of modern parallax measurements.

We use the Besancon stellar population model (Robin et al. 2003 ) to generate synthetic stars between $10 \mathrm{pc}$ and $20 \mathrm{pc}$ for the thin (dots) and thick (star symbol) disk populations at the galactic position of CFBDS0059. Figure 3 shows their proper motions together with those of CFBDS0059 and ULAS0034. The contour lines show the probability that an object with a given proper motion belongs to the thin disk rather than the thick disk (halo membership probabilities are negligible). CFBDS0059, at its probable distance, is well within the $95 \%$ probability thin disk membership region, and ULAS0034 is within the 99\% probability region. In spite of its somewhat high proper motion for an object beyond $10 \mathrm{pc}$, CFBDS0059 therefore most likely belongs to the thin disk. The mean age of the simulated stars in the region of the proper motion diagram occupied by CFBDS0059 is 4 Gyr, suggesting that it is an older member of the thin disk. That age is consistent with the 1 to 5 Gyr range derived below from comparison to COND evolutionary models (Baraffe et al. 2003). As any kinematic age for an individual star, this determination has large error bars, but it suggests that CFBDS0059 might be older than ULAS0034.

\section{Spectral comparison and atmospheric parameters}

Figures 4 and 5 present our spectrum of CFBDS0059, together with those of ULAS0034 (Warren et al. 2007, >T8), 2M0415 (Burgasser et al. 2003, T8) and G1570D (Burgasser et al. 2000, T7.5), which successively were the coolest known brown dwarfs. Thanks to their earlier discovery, 2M0415 and G1570D have the best characterized atmospheric parameters (Saumon et al. 2006, 2007), and they provide the most solid baseline for a differential study. The Warren et al. (2007) spectrum of ULAS0034, kindly communicated by Leggett, was obtained with GNIRS on Gemini South and its $\frac{\lambda}{\Delta \lambda}=500$ resolution matches that of our NIRI spectrum of CFBDS0059. We downloaded the 


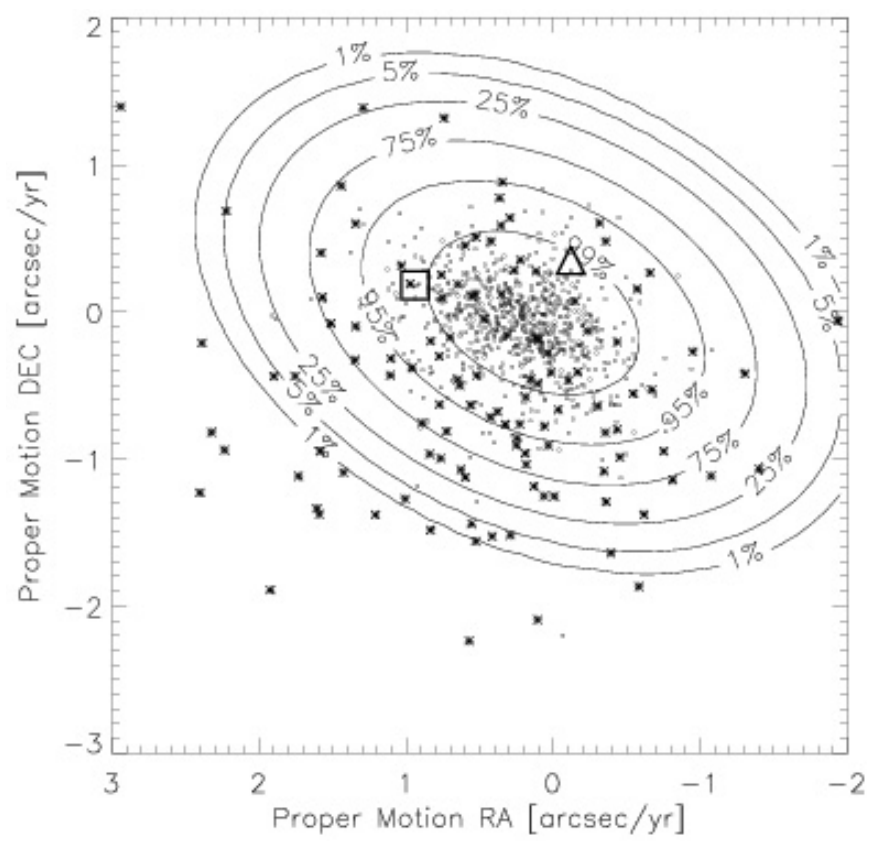

Fig. 3. Thin disk probability membership contours in proper motion space from the Besancon stellar population model. The contours are generated for synthetic stars with distances between 10 and $20 \mathrm{pc}$, belonging to the thin disk (small dots) and the thick disk (small stars, with the density of the latter increased by a factor of 10 for display purposes). Less than one halo star would appear on the plot. Based on their measured proper motion, the likelihood that CFBDS0059 (large open square) and ULAS0034 (large open triangle) belongs to the thin disk are $95 \%$ and $>99 \%$.

Burgasser et al. (2003, 2002) OSIRIS spectra of the two other brown dwarfs from the Ultracool Dwarf Catalog ${ }^{2}$, and degraded their original spectral resolution of $\frac{\lambda}{\Delta \lambda} \sim 1200$ to match that of the GNIRS and NIRI spectra. Stronger telluric absorption from the lower altitude telescopes explains the wider blanked regions in the corresponding spectra, but doesn't measurably affect any comparison: as illustrated by CFBDS0059, late-T dwarfs have essentially negligible flux wherever telluric $\mathrm{H}_{2} \mathrm{O}$ absorption matters. Because the OSIRIS spectra do not cover the $Y$ band, we complement them by lower resolution spectra from Geballe et al. (2001) and Knapp et al. (2004) for $\lambda<1.18 \mu \mathrm{m}$.

\subsection{Atmospheric parameters}

Atmospheric parameters of ultracool dwarfs are ideally determined from a combination of near and mid-IR information (e.g. Saumon et al. 2006, 2007), but low resolution near-infrared spectra alone provide a useful proxy when mid-IR photometry and spectra are not (yet) available (e.g. Burgasser et al. 2006a; Leggett et al. 2007). Burgasser et al. (2006a) used a grid of solar metallicity cool brown dwarfs to calibrate two spectral ratios, $\mathrm{H}_{2} \mathrm{O}-\mathrm{J}$ and $\mathrm{K} / \mathrm{H}$, which respectively measure the strength of $\mathrm{H}_{2} \mathrm{O}$ absorption at $\sim 1.15 \mu \mathrm{m}$ and the flux ratio between the $K$ and $H$ peaks, to $T_{\text {eff }}$ and $\log g$. Warren et al. (2007) however found that $\mathrm{H}_{2} \mathrm{O}-J$ essentially saturates below $T_{\text {eff }}=750 \mathrm{~K}$, and therefore chose not to use this spectral index for spectral types later than T8. They demonstrate on the other hand that the combination of the $K / J$ index with the width of $J$-band peak, parametrised by their $W_{J}$ index, becomes a good $T_{\text {eff }}$ and $\log g$

\footnotetext{
2 http://www.iac.es/galeria/ege/catalogo_espectral/ index.html
}

diagnostic at $T_{\text {eff }} \simeq 900 \mathrm{~K}$, and remains useful significantly below $750 \mathrm{~K}$. We adopt their method.

Table 3 lists our measurement of these two indices for CFBDS0059, and Fig. 6 compares them with the Warren et al. (2007) measurements for 2M0415, HD3651B, G1570D and ULAS0034. To derive $T_{\text {eff }}$ and $\log g$ from the indices we use model indices from solar-abundance BT-settl atmospheric models (Warren et al. 2007; Allard et al. 2003; Allard et al. 2008, in prep.). The model with $\mathrm{NH}_{3}$ at chemical equilibrium abundance clearly produces too much absorption in the blue side of the $H$-band, confirming the finding of Saumon et al. (2006, 2007) that non-equilibrium processes keep the $\mathrm{NH}_{3}$ partial pressure well below its equilibrium value. We then use models that keep the abundances of $\mathrm{NH}_{3}$ and $\mathrm{N}_{2}$ at a fixed value in all parts of the atmosphere where the reaction timescale exceeds the mixing timescale, which typically occurs at the 600-800 K temperature level. These "quenched" models agree much better with the observed band shape.

As a first order correction for the remaining imperfections of the theoretical spectra, the model indices are shifted into agreement with the measurements of $2 \mathrm{M} 0415$ at the $\left(T_{\text {eff }}=750 \mathrm{~K}\right.$; $\log g=5.00$ and $[\mathrm{M} / \mathrm{H}]=0)$ determined for that brown dwarf by Saumon et al. (2007). The $T_{\mathrm{eff}}=800 \mathrm{~K}$ and $\log g=5.35 \mathrm{re}-$ sulting from this calibration for G1570D are consistent with the $T_{\text {eff }}=800-820 \mathrm{~K}$ and $\log g=5.1-5.25$ derived by Saumon et al. (2006) from a complete spectral analysis. For HD3651B (T7.5, Luhman et al. 2007), $T_{\text {eff }}=820-890 \mathrm{~K}$ and $\sim \log g=5.1-5.3$ resulting from this calibration are roughly consistent with the $T_{\text {eff }}=780-840 \mathrm{~K}$ and $\log g=5.1-5.5$ derived by Liu et al. (2007).

CFBDS0059 and ULAS0034 have very similar $W_{J}$ indices, but the new brown dwarf has a significatively smaller $K / J$ index. Visual comparison of the two spectra (Fig. 4) confirms that CFBDS0059 does have a weaker $K$-band peak than any of the 3 comparison cool brown dwarfs. As widely discussed in the recent literature (e.g. Liu et al. 2007, Fig. 3 in Burgasser et al. 2006a, or Fig. 3 in Leggett et al. 2007), for a fixed metallicity a weaker $K$-band peak is evidence of either a lower temperature or a higher gravity. The $W_{J}$ index lifts this degeneracy: it indicates, again assuming identical chemical compositions for the two brown dwarfs, that CFBDS0059 is cooler by $\sim 50 \pm 15 \mathrm{~K}$ and has a $\sim 0.15 \pm 0.1$ higher $\log g$ than ULAS0034.

As also discussed by Warren et al. (2007), the above uncertainties only reflect the random errors in the spectral indices. They are appropriate when comparing two very similar objects, like CFBDS0059 and ULAS0034, since systematic errors then cancel out. They must on the other hand be increased to compute absolute effective temperatures and gravity: one then needs to account for the uncertainties on the 2M0415 parameters which anchor the Fig. 6 grid $(T \sim \pm 25 \mathrm{~K}$ and $\log g \sim \pm 0.2$; Saumon et al. 2007), and for the uncertainties in the atmospheric models which may distort the grid between its anchor point $\left(T_{\text {eff }}=750 \mathrm{~K} ; \log g=5.00\right.$ and $\left.[\mathrm{M} / \mathrm{H}]=0\right)$ and the $\sim 600 \mathrm{~K} \mathrm{re}-$ gion of interest here. We conservatively adopt $T_{\text {eff }}=620 \pm 50 \mathrm{~K}$ and $\log g=4.75 \pm 0.3$.

This 2-parameter analysis obviously cannot determine all three main atmospheric parameters ( $T_{\mathrm{eff}}, \log g$ and metallicity). As discussed by Warren et al. (2007), it actually determines the temperature with no ambiguity but leaves a combination of $[\mathrm{M} / \mathrm{H}]$ and $\log g$ undetermined, and they demonstrated that in the $W_{J}$ versus $J / K$ plot metallicity is degenerate with surface gravity, with $\Delta(\log g) \equiv-2 \Delta[\mathrm{M} / \mathrm{H}]$. CFBDS0059 is thus definitely cooler than ULAS0034, but from $W_{J}$ versus $J / K$ diagram it can have either higher surface gravity or lower 

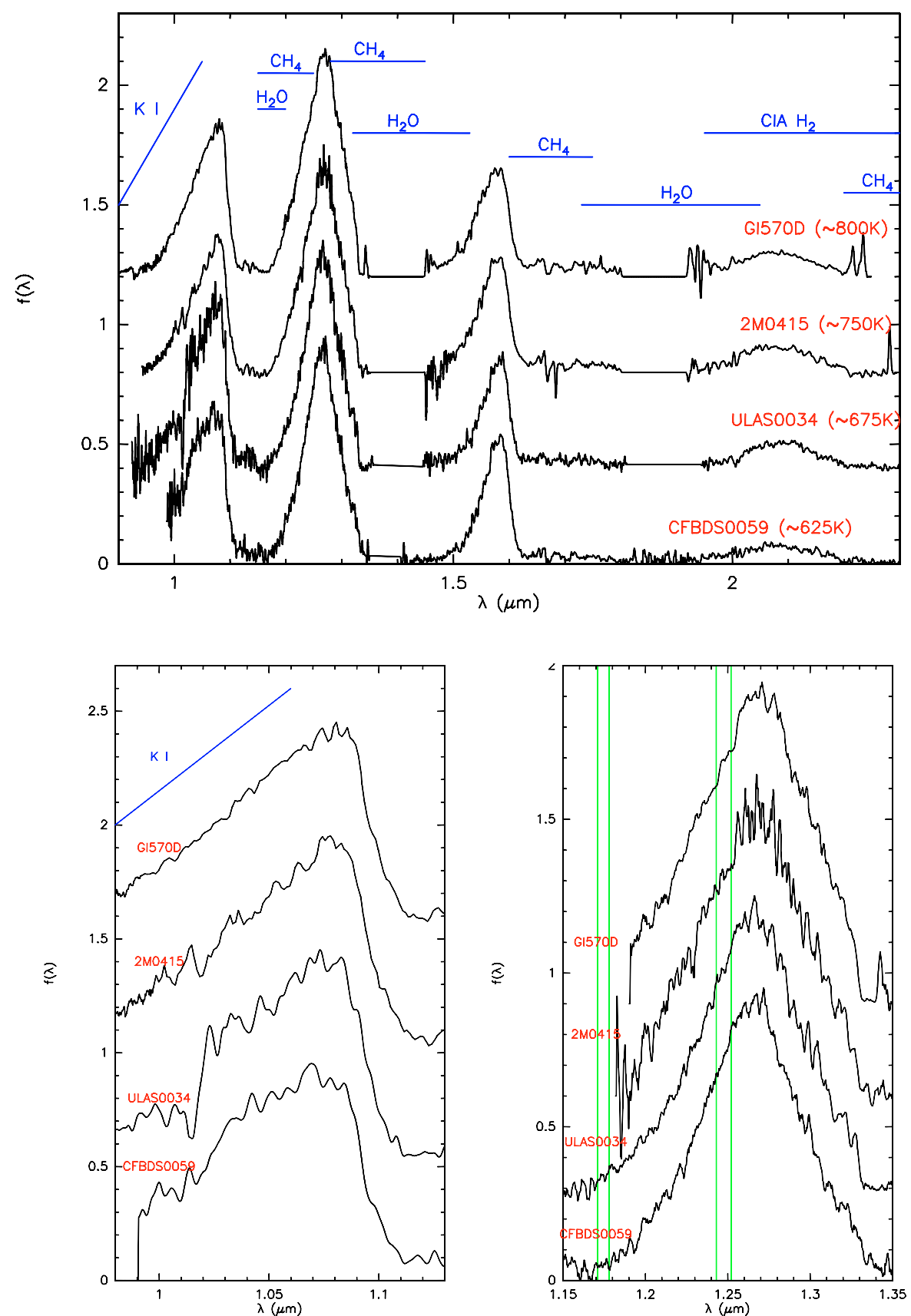

Fig. 4. 0.9-2.3 $\mu \mathrm{m}$ spectra of CFBDS0059 and the three other coolest brown dwarfs. The spectra are normalized to unit flux densities at their $1.27 \mu$ m.peak, and vertically offset for clarity. The main $T$-dwarf spectral features are labeled. The temperatures of $2 \mathrm{M} 0415$ and Gl570D are from the careful spectroscopic analyses of Saumon et al. (2006) and Saumon et al. (2007). Those of CFBDS0059 and ULAS0034 are from our $W_{J}$ versus $J / K$ index (Fig. 6).

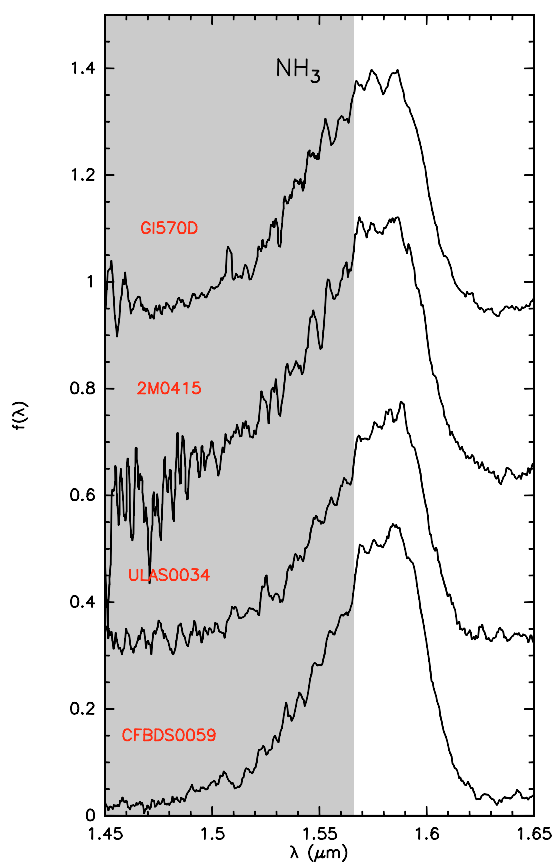

Fig. 5. $Y, J$ and $H$-bands spectra of the four cool brown dwarfs. The green vertical lines in the 1.15-1.35 $\mu$ m panel mark the two potassium doublets. The grey band in the $1.45-1.65 \mu \mathrm{m}$ panel indicates the approximate limits of the new absorption bands, which we discuss in the text.

Table 3. Measured spectral classification indices for CFBDS0059 and ULAS 0034.

\begin{tabular}{lllllll}
\hline $\begin{array}{l}\text { Spectral } \\
\text { indice }\end{array}$ & $\begin{array}{l}\text { Numerator } \\
\text { wavelength }\end{array}$ & $\begin{array}{l}\text { Denominator } \\
\text { wavelength } \\
\mu \mathrm{m}\end{array}$ & \multicolumn{2}{c}{$\begin{array}{l}\text { Value } \\
\text { CFBDS0059 }\end{array}$} & $\begin{array}{c}\text { Spectral } \\
\text { type }\end{array}$ & $\begin{array}{l}\text { Value } \\
\text { ULAS0034 }\end{array}$ \\
\hline $\mathrm{H}_{2} \mathrm{O}-J$ & $1.14-1.165$ & $1.26-1.285$ & $0.029 \pm 0.005$ & $\mathrm{~T} 8$ & $0.012 \pm 0.007$ & $>\mathrm{T} 8$ \\
$\mathrm{CH}_{4}-J$ & $1.315-1.34$ & $1.26-1.285$ & $0.165 \pm 0.005$ & $\mathrm{~T} 8$ & $0.014 \pm 0.009$ & $>\mathrm{T} 8$ \\
$\mathrm{H}_{2} \mathrm{O}-H$ & $1.48-1.52$ & $1.56-1.60$ & $0.119 \pm 0.008$ & $>\mathrm{T} 8$ & $0.133 \pm 0.010$ & $>\mathrm{T} 8$ \\
$\mathrm{CH}_{4}-H$ & $1.635-1.675$ & $1.56-1.60$ & $0.084 \pm 0.002$ & $\mathrm{~T} 8$ & $0.096 \pm 0.006$ & $\mathrm{~T} 8$ \\
$\mathrm{CH}_{4}-K$ & $2.215-2.255$ & $2.08-2.12$ & $0.128 \pm 0.037$ & $>\mathrm{T} 7$ & $0.091 \pm 0.015$ & $>\mathrm{T} 7$ \\
$K / J$ & $2.06-2.10$ & $1.25-1.29$ & $0.101 \pm 0.002$ & & $0.128 \pm 0.003$ & \\
$W_{J}$ & $1.18-1.23$ & $1.26-1.285$ & $0.257 \pm 0.004$ & & $0.262 \pm 0.006$ & \\
\hline
\end{tabular}




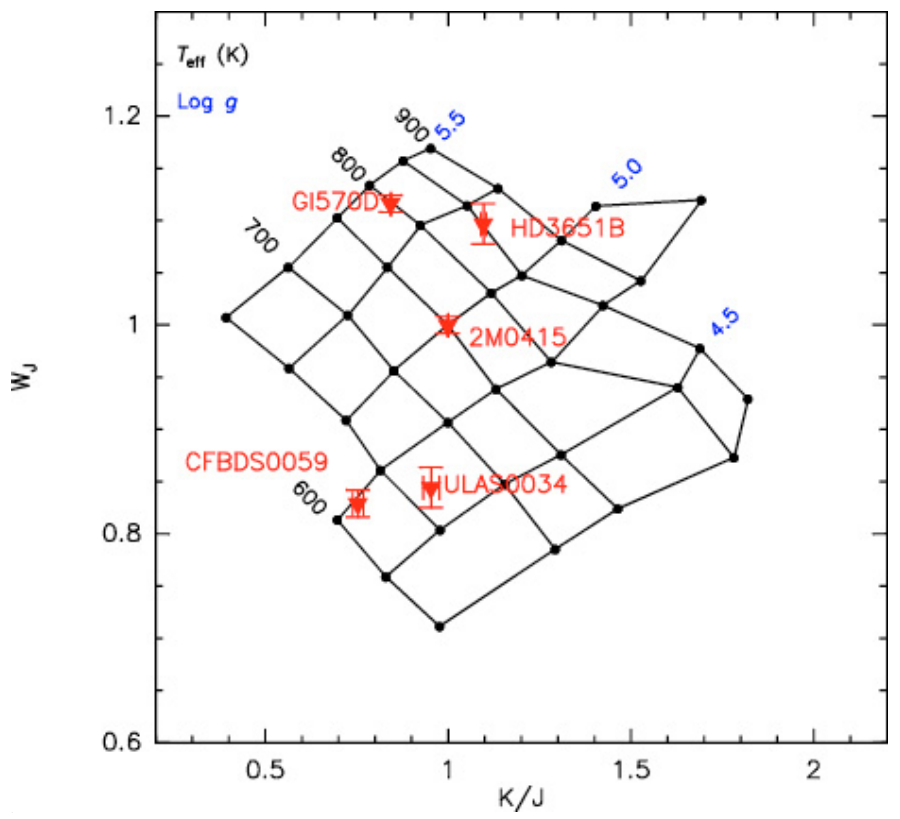

Fig. 6. $W_{J}$ versus $J / K$ indices. The grid represents indices measured on solar metallicity BT-settl model spectra, shifted into agreement of the $\left(T_{\text {eff }}=750 \mathrm{~K} ; \log g=5.00\right.$ and $\left.[\mathrm{M} / \mathrm{H}]=0\right)$ with the measured indices of $2 \mathrm{M} 0415$. The error bars represent the $1 \sigma$ uncertainties of the measured spectral indices.

metallicity. This degeneracy affects the full $J H K$-band spectrum, where any metallicity vs. gravity difference is at most very subtle. It is however lifted by the shape of the $Y$-band peak (Figs. 3 of Burgasser et al. 2006a or Leggett et al. 2007), since lower metallicity shifts the $Y$-band flux density peak of submetallic brown dwarfs significantly blueward. Figure 5 shows no such shift, and the two objects therefore have similar metallicities.

Figure 7 overlays the observed CFBDS0059 spectrum with the synthetic spectrum for the closest point of the solar metallicity atmospheric model grid. Except on the red side of the $H$-band, model and observations agree well, boosting our confidence in the derived atmospheric parameters. The main remaining predictive shortcoming of the models is their overestimated absorption on the red side of the $H$-band peak. The principal opacity source in this region is the methane band centred at $1.67 \mu \mathrm{m}$, for which comprehensive theoretical predictions are available, but only for transitions from the vibrational ground state (as will be discussed in detail in Homeier et al., in preparation). To make up for the missing absorption from higher bands, which constitutes a significant fraction of the opacity at brown dwarf temperatures, a constant empirical correction factor was used. This correction must in turn lead to some overestimate of the $\mathrm{CH}_{4}$ absorption as we reach the lower end of the $T$ dwarf temperature range. Another possible source of errors are uncertainties in the models' temperature profile. The BT-Settl models self-consistently describe gravitational settling, convective turbulence, advection, condensation, coalescence and coagulation of condensates to predict the formation and vertical extent of cloud layers (Allard et al. 2003; Helling et al. 2007). In late $T$ dwarfs these clouds are predicted to reside deep in the optically thick part of the atmosphere. Their opacity is thus not directly visible in the spectrum, but it may still impact the thermal structure, and thus the relative abundance especially of temperature-sensitive species like $\mathrm{CH}_{4}$.

Another (less serious) disagreement between the models and the observed spectra occurs in the $Y$ band. The models overestimate the flux on the blue side of the $Y$-peak, and they imperfectly reproduce the general shape of the peak. As discussed below, the opacities in that band are dominated by pressurebroadened wings of the $0.77 \mu \mathrm{m} \mathrm{K} \mathrm{I} \mathrm{line} \mathrm{on} \mathrm{the} \mathrm{blue} \mathrm{side} \mathrm{and}$ $\mathrm{CH}_{4}$ on the red side.

For Baraffe et al. (2003) evolutionary models, the $T_{\text {eff }} \simeq$ 570-670 K and $\log g \simeq 4.45-5.05$ determined above translate into an age of $1-5 \mathrm{Gyr}$ and a mass of $15 M_{\mathrm{Jup}}$ (for $1 \mathrm{Gyr}$ ) to $30 M_{\text {Jup }}$ (for $5 \mathrm{Gyr}$ ). The kinematics of CFBDS0059 suggests that it belongs to an older population, and therefore slightly favour a higher mass and older age.

\subsection{Individual spectral features}

Figure 5 zooms on the $Y, J$ and $H$-band peaks of the four cool brown dwarf spectra. The published OSIRIS spectra of 2M0415 and G1570D do not cover the $Y$ band, which instead is plotted from the lower resolution spectra of Geballe et al. (2001) and Knapp et al. (2004). For easier comparison, the CFBDS0059 and ULAS0034 $Y$-band spectra are smoothed to that resolution.

Direct comparison of the four spectra can be used to shed light on incipient new features and atmospheric chemistry. Features which are seen in both CFBDS0059 and ULAS0034 are likely to be real even when their significance is modest in each object, and those which are absent or weaker in the two hotter brown dwarfs, can reasonably be assigned to low temperature molecules. Conversely, features which disapear in the two cooler objects trace higher temperature species.

As discussed above, the $Y$-band spectra of CFBDS0059 and ULAS0034 do not differ much. Given the strong sensitivity of that band to $[\mathrm{M} / \mathrm{H}]$ that implies that the two objects have similar chemical compositions. The shape of the $Y$-band peaks of these two coolest brown dwarfs on the other hand differ from that of 2M0415 and G1570, with the CFBDS0059 and ULAS0034 peaks extending further into the blue. The dominant absorbers in the blue wings of the $Y$-band peak is the pressure-broadened wing of the $0.77 \mu \mathrm{m} \mathrm{K} \mathrm{I} \mathrm{line} \mathrm{(e.g.} \mathrm{Burgasser} \mathrm{et} \mathrm{al.} \mathrm{2006a),}$ which must weaken as $\mathrm{K}$ I depletes from the gas phase under $T_{\text {eff }}=\sim 700 \mathrm{~K}$. As anticipated by Leggett et al. (2007), the slope of the blue side of the $Y$-band peak therefore shows good potential as an effective temperature diagnostics beyond spectral type T8.

The strength of the $J$-band K I doublet is a good gravity estimator in ultracool dwarfs (e.g. Knapp et al. 2004), because an increased pressure at a fixed temperature favors $\mathrm{KCl}$ over K (Lodders 1999) and consequently weakens atomic potassium features. At $T_{\text {eff }} \sim 750-800 \mathrm{~K}$ the the $J$-band $\mathrm{K}$ I doublets remain weakly visible and useful as a gravity proxy (Fig. 7 of Knapp et al. (2004)). At $T_{\text {eff }}<700 \mathrm{~K}$ on the other hand, the $\mathrm{K}$ I doublets have completely vanished at the resolution of the current spectra (Fig. 5), even at the probably lower gravity of ULAS0034. Potassium is thus mostly converted to $\mathrm{KCl}$ (or perhaps other compounds) in the relevant photospheric layers.

The strongest new feature is wide absorption on the blue side of the $H$-band, at $\lambda<1.565 \mu \mathrm{m}$. It is conspicuous in CFBDS0059 and well detected in ULAS0034, and with hindsight is weakly visible in the $2 \mathrm{M} 0415$ spectrum (Fig. 5). It is however clearly stronger at $T_{\text {eff }}<700 \mathrm{~K}$. To visually emphasize this broad feature, we bin the spectra to $R=\sim 100$ and overlay the four H-band spectra (Fig. 8, left panel). Absorption sets in at $\sim 1.585 \mu \mathrm{m}$ and becomes deeper for $\lambda<1.565 \mu \mathrm{m}$. These wavelengths overlap with strong $\mathrm{H}_{2} \mathrm{O}$ and $\mathrm{NH}_{3}$ bands. Either molecule could a priori be responsible for the absorption. 


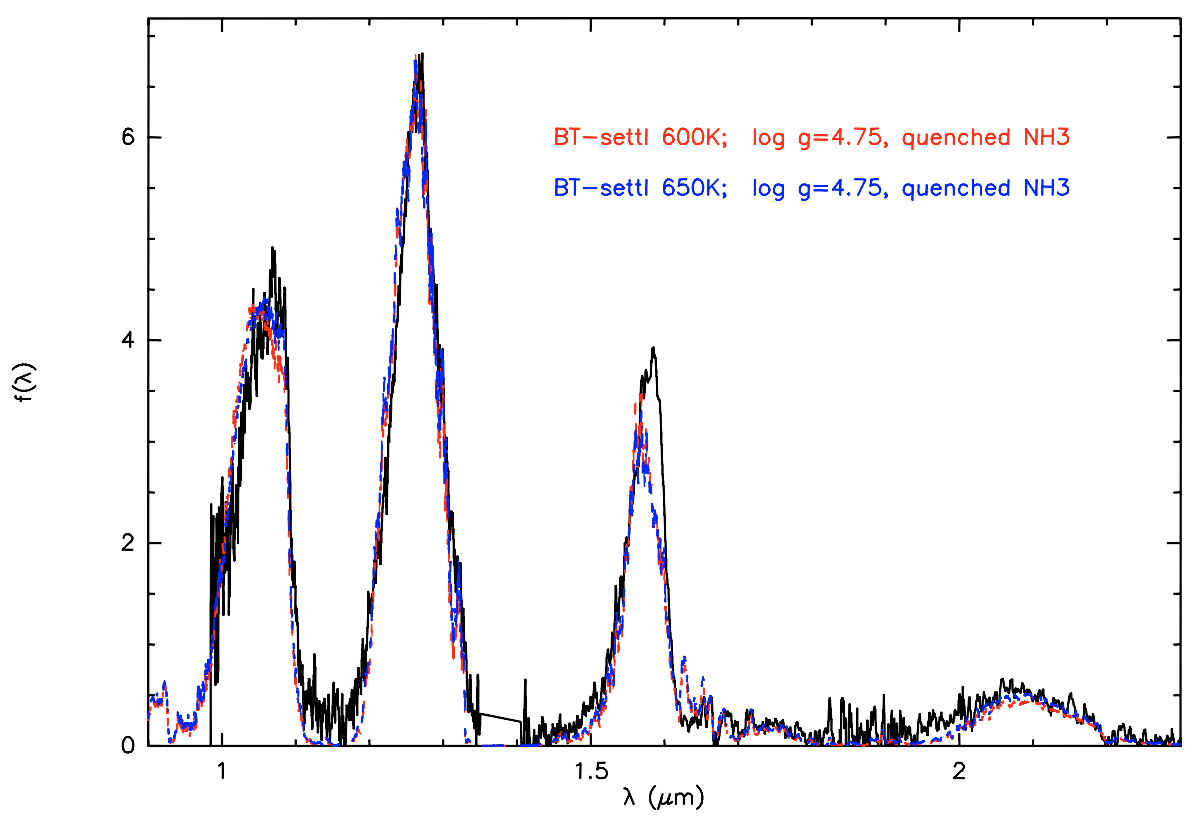

Fig. 7. Overlay of the CFBDS0059 spectrum with the solar metallicity $\left(T_{\text {eff }}=600-650 \mathrm{~K}\right.$; $\log g=4.75)$ BT-settl synthetic spectrum. The two spectra are scaled to agree at their $1.27 \mu \mathrm{m}$ flux peak. The "quenched NH3" models are chemical equilibrium models which enforce a constant abundance of ammonia in the cooler regions of the atmosphere.
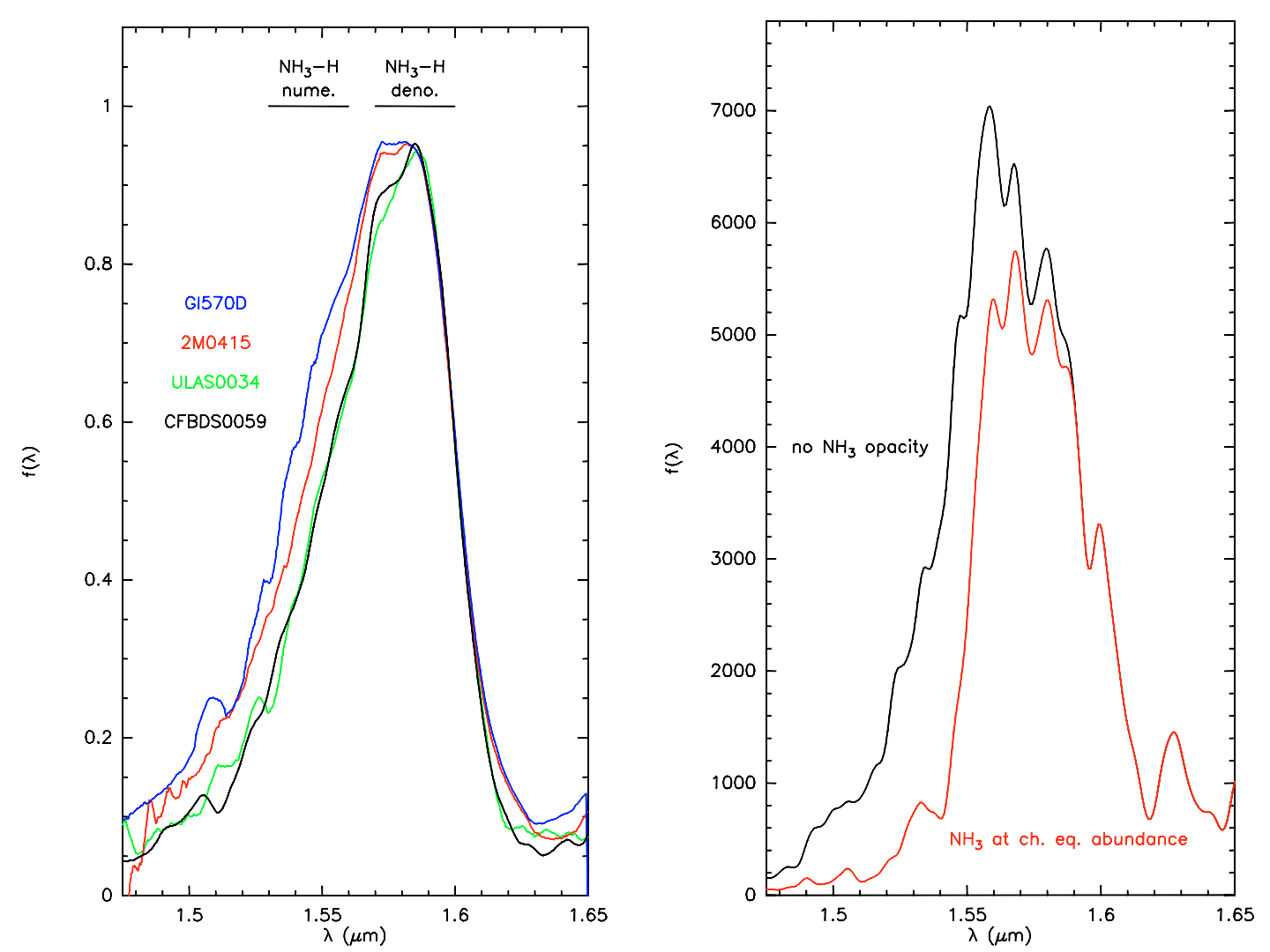

Fig. 8. Left: $H$-band spectrum of the four cool brown dwarfs binned to $R=\sim 100$. The spectra are normalized at $\lambda=1.59 \mu \mathrm{m}$. The integration intervals for the numerator and denominator of our proposed $\mathrm{NH}_{3}-H$ index are marked. Right: BT-Settl synthetic spectra for $\left(T_{\text {eff }}=600 \mathrm{~K}\right.$; $\log g=4.75$ ) with and without near-infrared $\mathrm{NH}_{3}$ opacity; the $\mathrm{NH}_{3}$ abundance is at its chemical equilibrium value.

\subsection{Near infrared ammonia signatures}

Those molecules unfortunately have imperfect opacity data, and the $\mathrm{NH}_{3}$ laboratory line lists in particular are incomplete below $1.7 \mu \mathrm{m}$. Computed ammonia opacities are therefore strictly lower limits. Leggett et al. (2007) compare synthetic spectra computed with and without $\mathrm{NH}_{3}$ opacity, using the
Irwin et al. (1999) line list for $\lambda<1.9 \mu \mathrm{m}$, and find that ammonia absorption in cold brown dwarfs strongly depletes the blue wing of the $H$ band (their Fig. 10). Similarly, Fig. 9 of Saumon et al. (2000) plots synthetic $H$-band spectra with and without $\mathrm{NH}_{3}$ opacity, and find differences in two wavelength ranges: the $\mathrm{NH}_{3}$-rich model is significantly more absorbed for 

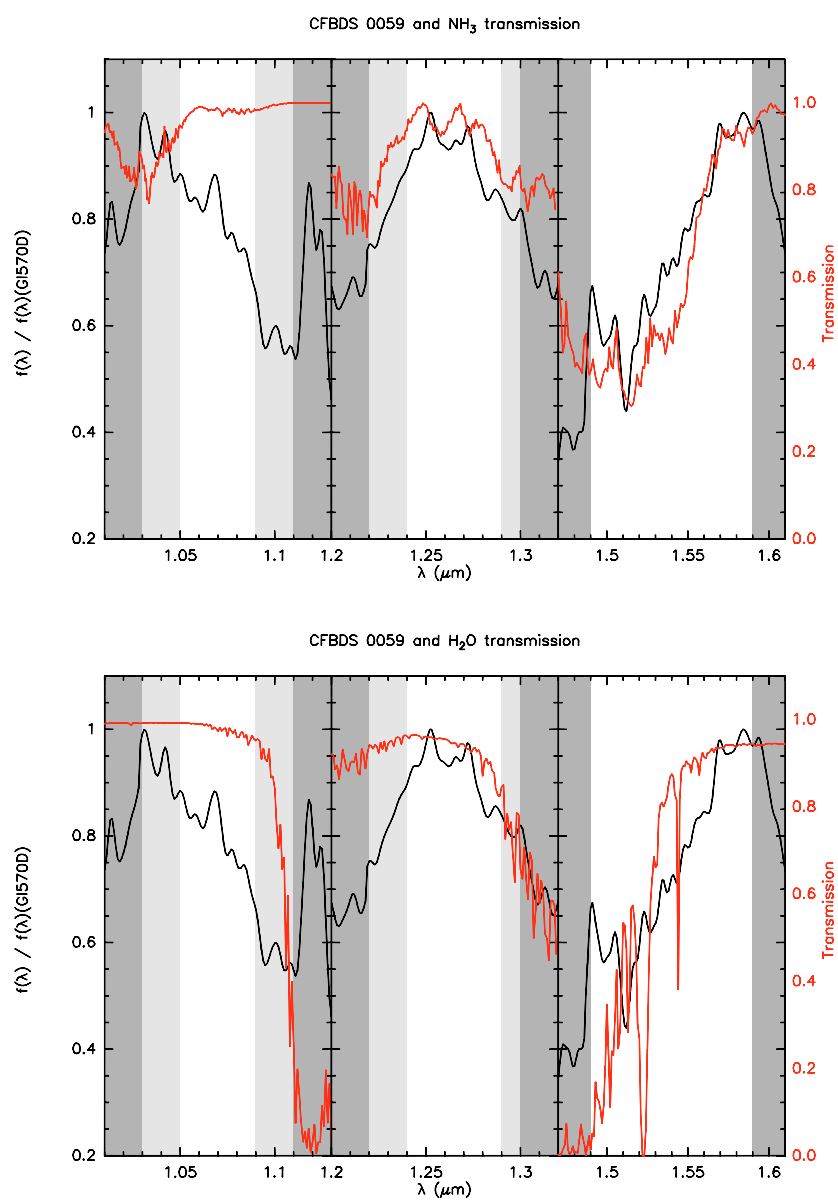

Fig. 9. Flux ratio between CFBDS0059 and G1570D (black), together with the laboratory room temperature transmission spectrum of $\mathrm{NH}_{3}$ (Irwin et al. 1999) (red, top panel) and the $600 \mathrm{~K} \mathrm{H}_{2} \mathrm{O}$ transmission spectrum computed from the HITRAN molecular database (red, bottom panel). The grey bands mark the parts of the spectrum affected by strong (dark grey) or moderate (light grey) $\mathrm{CH}_{4}$ absorption.

$\lambda<1.565 \mu \mathrm{m}$ and it has weaker but significant absorption in the $(1.5725-1.585 \mu \mathrm{m})$ range.

Figure 8 right panel plots two BT-Settl models for $\left(T_{\text {eff }}=\right.$ $600 \mathrm{~K} ; \log g=4.75$ ), without any near-infrared $\mathrm{NH}_{3}$ opacity, and with $\mathrm{NH}_{3}$ opacity for that molecule at its chemical equilibrium abundance. As discussed above the BT-Settl models do not reproduce the observed $H$-peak shape very well, and a quantitative comparison is thus difficult. The comparison of the two models nonetheless confirms the Saumon et al. (2000) conclusion that ammonia produces strong absorption below $\sim 1.57 \mu \mathrm{m}$ and weaker residual out to $1.595 \mu \mathrm{m}$. These model predictions qualitatively match the behaviour seen in Fig. 8, left panel.

To emphasize the changes in brown dwarfs spectra when their effective temperature decreases from $\sim 800$ to $\sim 600 \mathrm{~K}$, we plot in Fig. 9 the ratio of the spectra of CFBDS0059 and G1570D. The signal to noise ratio of the resulting $K$-band spectrum is too low for detailed analysis, and we therefore focus on the $Y$, $J$ and $H$ flux peaks. To avoid confusion from changes in the temperature-sensitive methane bands, we also mostly ignore the parts of the spectrum affected by $\mathrm{CH}_{4}$ absorption bands, hatched in dark and light grey for respectively stronger and weaker bands. Figure 10 shows the equivalent plot for ULAS0034, which is very similar.
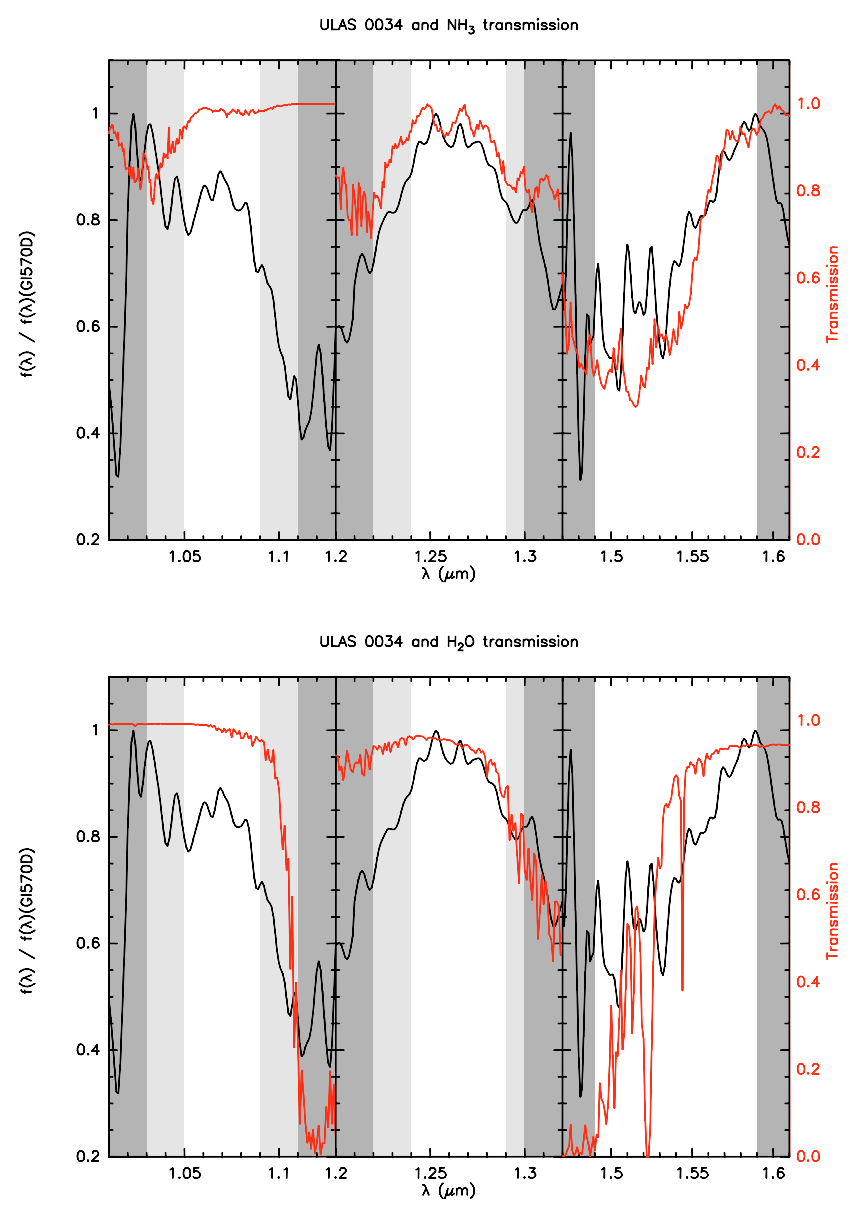

Fig. 10. Flux ratio between ULAS0034 and Gl 570D (black). The overlays repeat those of Fig. 9.

The $H$-band spectrum ratio prominently shows the new absorption band, which outside the $\mathrm{CH}_{4}$ band closely matches the $300 \mathrm{~K} \mathrm{NH}_{3}$ transmission spectrum of Irwin et al. (1999). Both spectra are strongly absorbed between 1.49 and $1.52 \mu \mathrm{m}$ and rebound from 1.52 to $1.57 \mu \mathrm{m}$. Water absorption, by contrast, is a poor match to the features of spectrum ratio. The strongest water absorption (as computed from the HITRAN molecular database for a $600 \mathrm{~K}$ temperature) occurs below $1.49 \mu \mathrm{m}$, at significantly bluer wavelengths than the CFBDS0059 absorption feature.

Some weaker but still significant bands of the Irwin et al. (1999) laboratory ammonia spectrum occur in the $J$ band. Those again match features of the CFBDS0059/G1570D flux ratio, but that agreement is much less conclusive: water and ammonia absorptions overlap on the red side $J$-band peak, and $\mathrm{CH}_{4}$ absorption affects the blue side of the peak. A $1.25-1.27 \mu \mathrm{m}$ feature is seen on both flux ratios and on the ammonia transmission, and could be due to ammonia since it is clear of any strong $\mathrm{H}_{2} \mathrm{O}$ absorption band. The slight wavelength shift between the laboratory and observed features however leaves that association uncertain. Detailed synthetic spectra based on fully reliable opacities will be needed to decide whether $\mathrm{NH}_{3}$ absorption matters in the $J$ band at the effective temperature of CFBDS0059. The main pattern in the $Y$-band is a blue slope, which reflects the weaker pressure-broadened $\mathrm{K}$ I wing in the cooler brown dwarf. The weak $1.03 \mu \mathrm{m} \mathrm{NH}_{3}$ band is not seen.

Ammonia is easily detected in mid-infrared SPITZER spectra for all spectral types cooler than T2 (Roellig et al. 2004; 
Cushing et al. 2006), though significantly weaker than initially expected because mixing from lower atmospheric levels reduces its abundance in the high atmosphere below the local equilibrium value (Saumon et al. 2006). Weak near-infrared absorption by ammonia has been tentatively detected by Saumon et al. (2000) in the T7p dwarfs G1 229B, but CFBDS0059 and ULAS0034 provide the first incontrovertible evidence of a strong nearinfrared $\mathrm{NH}_{3}$ band in brown dwarf spectra.

This conclusion contrasts with Warren et al. (2007) finding possible but inconclusive evidence of ammonia in ULAS0034. The main difference between the two analyses is that Warren et al. (2007) focused on a higher resolution search, at a necessarily lower signal to noise ratio, for individual $\mathrm{NH}_{3}$ lines between 1.5 and $1.58 \mu \mathrm{m}$. We instead looked for the global signature of the absorption band, which only becomes obvious when looking at the full $H$-band spectrum.

\subsection{Spectral type}

Table 3 lists for CFBDS0059 the spectral indices used by the spectral classification scheme of Burgasser et al. (2006b), which refines the previous schemes of Geballe et al. (2002) and Burgasser et al. (2002). These indices would imply a T8 classification, identical to that of 2M0415. As discussed above however, the near-infrared spectrum of CFBDS0059 demonstrates that it is over $100 \mathrm{~K}$ cooler than $2 \mathrm{M} 0415$ and shows clearly different spectral features. Based on the new indices we present later, CFBDS0059 should be assigned a later spectral type. The almost identical Burgasser et al. (2006b) indices of the two brown dwarfs instead reflect those indices measuring $\mathrm{H}_{2} \mathrm{O}$ and $\mathrm{CH}_{4}$ absorption bands which saturate and lose their effective temperature sensitivity at the T8 spectral type of 2M0415. Beyond T8 the Burgasser et al. (2006b) classification scheme therefore needs to be extended, with new spectral indicators that do not saturate until significantly later spectral types.

Fully defining this extension is beyond the scope of the present paper, since two known objects beyond T8 are not enough to explore spectral variability, but one can nonetheless start exploring. Since the main new feature is $\mathrm{NH}_{3}$ absorption in the blue wings of the $H$-band peak, we define a new $\mathrm{NH}_{3}-H$ index as

$\mathrm{NH}_{3}-H=\frac{\int_{1.53}^{1.56} f(\lambda) \mathrm{d} \lambda}{\int_{1.57}^{1.60} f(\lambda) \mathrm{d} \lambda}$.

Its numerator and denominator range are plotted in Fig. 8. The numerator integrates the flux within the main $\mathrm{NH}_{3}$ band and its denominator measures the bulk of the $H$-band peak (we note that in cooler objects, the denominator could be affected by some $\mathrm{NH}_{3}$ absorption; its integration boundaries might thus need to be refined after such objects have been discovered). We compute this index for G1 570D, HD3651B, 2M0415, ULAS0034 and CFBDS0059 (Table 4), and find that it strongly decreases from G1570D to ULAS0034 and CFBDS0059 (which have very similar $\mathrm{NH}_{3}-H$ ).

Over the limited effective temperature range spanned by G1570D, HD3651B, 2M0415, ULAS0034 and CFBDS0059, and as far as one can infer from just 5 examples, the $\mathrm{NH}_{3}-\mathrm{H}$ and $W_{J}$ indices correlate strongly (Fig. 11). The numerator of $W_{J}$ is centred at wavelengths where both ammonia (Fig. 10 of Leggett et al. 2007) and $\mathrm{CH}_{4}$ have significant opacity, and future modeling work should be able to establish whether the two indices probe the same molecule or not. Since the near-infrared spectra of ULAS0034 and CFBDS0059 differ significantly more from

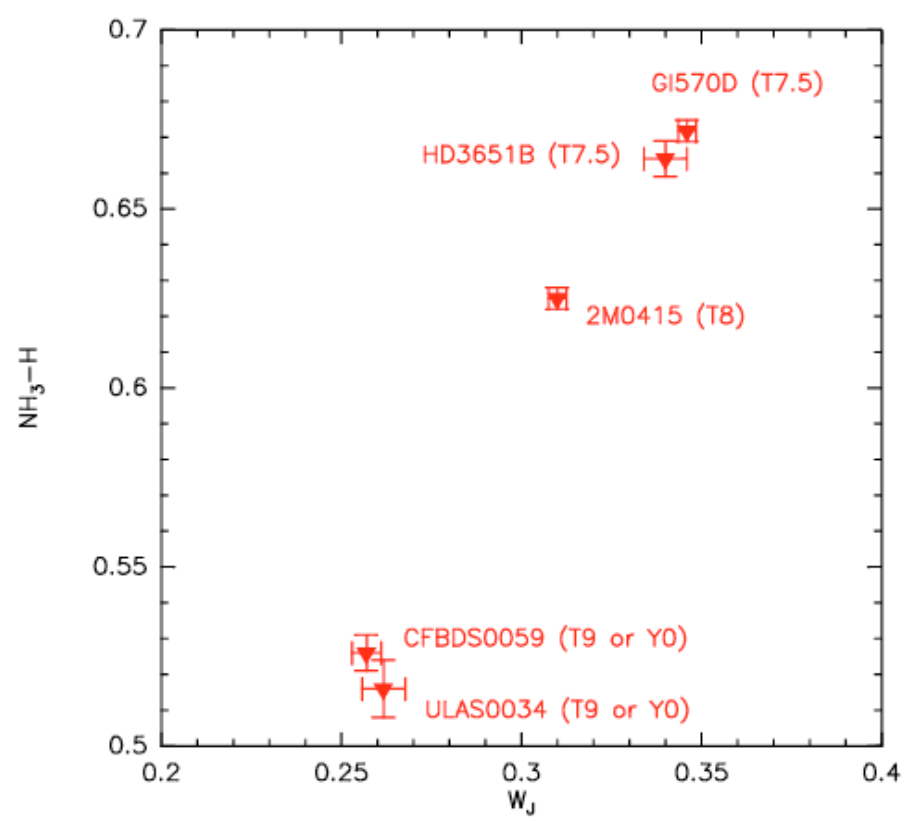

Fig. 11. $\mathrm{NH}_{3}-H$ index versus $W_{J}$ index. The error bars represent the $1 \sigma$ uncertainties of the measured spectral indices.

that of the T8 $2 \mathrm{M} 0415$ than the latter differs from the T7.5 G1570D (as quantitatively demonstrated by Fig. 11), it is natural to assign a full spectral subtype to the interval between the two coolest brown dwarfs and 2M0415. By that reasoning, and if ULAS0034 and CFBDS0059 are considered as $T$ dwarfs, their spectral type should be T9, or perhaps slightly later.

The $T$ spectral class however is quite unlikely to remain the last spectral type, since for sufficiently low effective temperatures atmospheric models predict major changes in visible and near-infrared brown dwarf spectra: $\mathrm{NH}_{3}$ bands are predicted to appear in, and eventually to dominate, the near-infrared spectrum, the strong pressure-broadened optical lines of $\mathrm{Na}$ I and $\mathrm{K} \mathrm{I}$ are predicted to disappear as those atomic species get incorporated into molecules and solids, and water clouds are predicted to form and to largely deplete water from the gas phase (Burrows et al. 2003; Kirkpatrick 2005). Since spectral classification, for mostly practical reasons, is traditionally based on optical and near-infrared spectra, such a major transition will justify the introduction of a new spectral type, for which the Y letter has long been reserved (Kirkpatrick et al. 1999; Kirkpatrick 2000). If the $\lambda \sim 1.55 \mu \mathrm{m} \mathrm{NH}_{3}$ band keeps deepening as the effective temperature decreases further, and eventually becomes a major spectral feature, its appearance at $T_{\text {eff }} \simeq 650 \mathrm{~K}$ will become a natural transition between the T and Y spectral classes. ULAS0034 and CFBDS0059 would then be the first Y dwarfs, and the prototypes for Y0 brown dwarfs, rather than T9. That decision will to some extent remain a matter of convention, but it must in any case wait until larger numbers of similarly cool brown dwarfs can document spectral trends in finer detail, and preferably over a wider effective temperature range.

\section{Summary and conclusions}

We have reported the discovery of CFBDS0059, a very cool brown dwarf, discovered in the CFBDS survey (Delorme et al. 2008). Its effective temperature is $\sim 50 \pm 15 \mathrm{~K}$ cooler than that of ULAS0034, most likely making it the coolest brown dwarf known at the present time. High spatial resolution imaging 
Table 4. $\mathrm{NH}_{3}-\mathrm{H}$ indices for the five brown dwarfs discussed in this paper.

\begin{tabular}{llllll}
\hline \hline & G1570D & HD3651B & 2M0415 & ULAS0034 & CFBDS0059 \\
\hline $\mathrm{NH}_{3}-H$ & $0.672 \pm 0.008$ & $0.66 \pm 0.005$ & $0.625 \pm 0.003$ & $0.516 \pm 0.008$ & $0.526 \pm 0.005$ \\
Spec. type & $\mathrm{T}^{2} .5^{1}$ & $\mathrm{~T}^{1} .5^{1}$ & $\mathrm{~T}^{1}$ & T9 or Y0 & T9 or Y0 \\
\hline
\end{tabular}

${ }^{1}$ Spectral classification follows Burgasser et al. (2006b). ${ }^{2}$ Spectral classification is derived from the $\mathrm{NH}_{3}-H$ and $W_{J}$ indices discussed in the text.

establishes than CFBDS0059 has no similarly bright companion beyond $0.09^{\prime \prime}$, and no companion with a contrast under $3.5 \mathrm{mag}$ beyond $0.3^{\prime \prime}$ (respectively 1.2 and 3.9 AU at the 13 pc photometric distance). Its kinematics suggest, with significant error bars, a 4 Gyr age at which CFBDS0059 would be a $\sim 30 M_{\text {Jup }}$ brown dwarf. The atmospheric parameters of CFBDS0059 however are compatible with any age from 5 Gyr down to $1 \mathrm{Gyr}$, for which its mass would be $\sim 15 M_{\text {Jup }}$. A trigonometric parallax measurement together with mid-infrared photometry and spectroscopy with SPITZER will significantly refine its physical parameters, as demonstrated by Saumon et al. (2007) for slightly warmer brown dwarfs.

We assign absorption in the blue wing of the $H$-band peaks of both ULAS 0034 and CFBDS 0059 to an $\mathrm{NH}_{3}$ band. If that assignment is confirmed, and if, as we expect, the band deepens at still lower effective temperatures, its development would naturally define the scale of the proposed $Y$ spectral class. ULAS 0034 and CFBDS 0059 would then become the prototypes of the Y0 sub-class.

The CFBDS survey has to date identified two brown dwarfs later than T8, CFBDS0059 and ULAS0034 (which we identified independently of Warren et al. 2007; Delorme et al. 2008) in the analysis of approximately $40 \%$ of its final 1000 square degree coverage. We therefore expect to find another few similarly cool objects, and hopefully one significantly cooler one.

CFBDS0059 and ULAS0034 provide a peek into the atmospheric physics for conditions that start approaching those in giant planets, and the future discoveries that can be expected from CFBDS, ULAS, and Pan-STARRS will further close the remaining gap. They also bring into a sharper light the remaining imperfections of the atmopsheric models, and emphasize in particular the importance of more complete opacity data. Our analysis relies on a room temperature absorption $\mathrm{NH}_{3}$ spectrum, but higher excitation bands than can be excited at $300 \mathrm{~K}$ must matter in $T_{\text {eff }}=600 \mathrm{~K}$ brown dwarfs. The eventual identification of ammonia absorption in the $J$ band will also need complete opacity information for $\mathrm{H}_{2} \mathrm{O}$ and $\mathrm{CH}_{4}$ and full spectral synthesis, since the bands of the three molecules overlap in that spectral range.

The spectral indices that define the $T$ dwarf spectral class saturate below $700 \mathrm{~K}$, which means that new ones will be needed at lower effective temperatures. We introduce one here, $\mathrm{NH}_{3}-\mathrm{H}$, which measures the likely $\mathrm{NH}_{3}$ absorption in the $H$ band. Together with the $W_{J}$ index of Warren et al. (2007) and the slope of the blue side of the $Y$-band peak (Leggett et al. 2007), it will hopefully define a good effective temperature sequence. Metallicity and gravity diagnostics are less immediately apparent, but will need to be identified as well.

Acknowledgements. We are grateful to our referee, Sandy Leggett, for her very detailed report and numerous suggestions which significantly improved this paper. We would like to thank the observers and queue coordinators who carried out our service observations at CFHT (programs 05BC05, 06AC20, 07BD97) and Gemini-North (GN-2007A-Q-201, GN-2007B-Q-3). We also thanks the NTT and Keck Observatory support astronomers for their help during the observations which led to these results. We thank S. Leggett and S Warren for providing their spectrum of ULAS0034 in a convenient numerical format, and Sandy Leggett for communicating spectra of 2M0415 and G1 570D and the room temperature absorption spectrum of $\mathrm{NH}_{3}$. We would also like to thank David Ehrenreich for providing us the computed $600 \mathrm{~K}$ water absorption. Financial support from the "Programme National de Physique Stellaire" (PNPS) of CNRS/INSU, France, is gratefully acknowledged. MCL acknowledges support for this work from NSF grant AST-0507833 and an Alfred P. Sloan Research Fellowship.

\section{References}

Allard, F., Guillot, T., Ludwig, H.-G., et al. 2003, in Brown Dwarfs, ed. E. Martín, IAU Symp., 211, 325

Baraffe, I., Chabrier, G., Barman, T. S., Allard, F., \& Hauschildt, P. H. 2003, A\&A, 402, 701

Bertin, E., \& Arnouts, S. 1996, A\&AS, 117, 393

Boulade, O., Charlot, X., Abbon, P., et al. 2003, in Instrument Design and Performance for Optical/Infrared Ground-based Telescopes, ed. M. Iye, \& A. F. M. Moorwood, Proc. SPIE, 4841, 72

Burgasser, A. J., Kirkpatrick, J. D., Cutri, R. M., et al. 2000, ApJ, 531, L57 Burgasser, A. J., Kirkpatrick, J. D., Brown, M. E., et al. 2002, ApJ, 564, 421 Burgasser, A. J., McElwain, M. W., \& Kirkpatrick, J. D. 2003, AJ, 126, 2487 Burgasser, A. J., Burrows, A., \& Kirkpatrick, J. D. 2006a, ApJ, 639, 1095 Burgasser, A. J., Geballe, T. R., Leggett, S. K., Kirkpatrick, J. D., \& Golimowski, D. A. 2006b, ApJ, 637, 1067

Burrows, A., Sudarsky, D., \& Lunine, J. I. 2003, ApJ, 596, 587 Chabrier, G., Baraffe, I., Allard, F., \& Hauschildt, P. 2000, ApJ, 542, 464 Cushing, M. C., Roellig, T. L., Marley, M. S., et al. 2006, ApJ, 648, 614 Delorme, P., Willott, C. J., Forveille, T., et al. 2008, A\&A, in press Devillard, N. 1997, The Messenger, 87, 19

Geballe, T. R., Saumon, D., Leggett, S. K., et al. 2001, ApJ, 556, 373 Geballe, T. R., Knapp, G. R., Leggett, S. K., et al. 2002, ApJ, 564, 466 Helling, C., Ackerman, A., Allard, F., et al. 2007, ArXiv e-prints, 711 Hodapp, K. W., Jensen, J. B., Irwin, E. M., et al. 2003, PASP, 115, 1388 Irwin, P. G. J., Calcutt, S. B., Sihra, K., et al. 1999, J. Quant. Spectr. Radiat. Transf., 62, 193

Kirkpatrick, J. D. 2000, in From Giant Planets to Cool Stars, ed. C. A. Griffith, \& M. S. Marley, ASP Conf. Ser., 212, 20

Kirkpatrick, J. D. 2005, ARA\&A, 43, 195

Kirkpatrick, J. D., Reid, I. N., Liebert, J., et al. 1999, ApJ, 519, 802

Knapp, G. R., Leggett, S. K., Fan, X., et al. 2004, AJ, 127, 3553

Leggett, S. K., Marley, M. S., Freedman, R., et al. 2007, ApJ, 667, 537

Liu, M. C., Leggett, S. K., \& Chiu, K. 2007, ApJ, 660, 1507

Lodders, K. 1999, ApJ, 519, 793

Luhman, K. L., Patten, B. M., Marengo, M., et al. 2007, ApJ, 654, 570

Monet, D. G., Levine, S. E., Canzian, B., et al. 2003, AJ, 125, 984

Pravdo, S. H., Shaklan, S. B., Wiktorowicz, S. J., et al. 2006, ApJ, 649, 389

Puget, P., Stadler, E., Doyon, R., et al. 2004, in Ground-based Instrumentation for Astronomy, ed. A. F. M. Moorwood, \& I. Masanori, Proc. SPIE, 5492, 978

Robin, A. C., Reylé, C., Derrière, S., \& Picaud, S. 2003, A\&A, 409, 523 Roellig, T. L., Van Cleve, J. E., Sloan, G. C., et al. 2004, ApJS, 154, 418 Saumon, D., Geballe, T. R., Leggett, S. K., et al. 2000, ApJ, 541, 374

Saumon, D., Marley, M. S., Cushing, M. C., et al. 2006, ApJ, 647, 552 Saumon, D., Marley, M. S., Leggett, S. K., et al. 2007, ApJ, 656, 1136 Scholz, R.-D., McCaughrean, M. J., Lodieu, N., \& Kuhlbrodt, B. 2003, A\&A, 398, L29

Simons, D. A., \& Tokunaga, A. 2002, PASP, 114, 169

Stephens, D. C., \& Leggett, S. K. 2004, PASP, 116, 9

Tokunaga, A. T., \& Vacca, W. D. 2005, PASP, 117, 421

Tokunaga, A. T., Simons, D. A., \& Vacca, W. D. 2002, PASP, 114, 180

van Dam, M. A., Bouchez, A. H., Le Mignant, D., et al. 2006, PASP, 118, 310

Warren, S. J., Mortlock, D. J., Leggett, S. K., et al. 2007, MNRAS, 381, 1400

Willott, C. J., Delorme, P., Omont, A., et al. 2007, AJ, 134, 2435

Wizinowich, P. L., Le Mignant, D., Bouchez, A. H., et al. 2006, PASP, 118, 297 